\title{
Increase in diastolic blood pressure induced by fragrance inhalation of grapefruit essential oil is positively correlated with muscle sympathetic nerve activity
}

\author{
Eriko Kawai ${ }^{1}$, Ryosuke Takeda ${ }^{2}$, Akemi Ota ${ }^{1}$, Emiko Morita ${ }^{1}$, Daiki Imai ${ }^{1,2}$, Yuta Suzuki ${ }^{1,2}$, Hisayo Yokoyama ${ }^{1,2}$, \\ Shin-ya Ueda ${ }^{3}$, Hidehiro Nakahara ${ }^{4}$,Tadayoshi Miyamoto ${ }^{5}$ and Kazunobu Okazaki ${ }^{1,2^{*}}$
}

\begin{abstract}
Fragrance inhalation of essential oils is widely used in aromatherapy, and it is known to affect blood pressure (BP) and heart rate (HR) via autonomic control of circulation. In this study, we aimed to test the hypothesis that the changes in hemodynamics with fragrance inhalation were observed along with changes in muscle sympathetic nerve activity (MSNA). In study 1, thirteen healthy men were exposed to fragrance stimulation of grapefruit essential oil for 10 min, and BP, HR, and MSNA were continuously measured. In study 2, another nine healthy men were exposed to the same fragrance stimulation; responses in BP and HR were continuously measured, and plasma noradrenaline and cortisol concentrations were determined. We found that diastolic BP increased significantly during fragrance inhalation, while the other variables remained unchanged in both studies. Although MSNA burst frequency, burst incidence, and total activity remained unchanged during fragrance inhalation, we found a significant linear correlation between changes in diastolic BP in the last 5 min of fragrance inhalation and changes in MSNA burst frequency. The plasma cortisol concentration decreased significantly at 10 min of fragrance inhalation, though the noradrenaline concentration remained unchanged. These results suggest, for the first time, that changes in BP with fragrance inhalation of essential oil are associated with changes in MSNA even with decreased stress hormone.
\end{abstract}

Keywords: Blood pressure, Autonomic nerve, Aromatherapy

\section{Introduction}

Aromatherapy is widely used for variety of purposes [1-4]. Generally, essential oils extracted from plants or fruits are used for aromatherapy as the fundamental fragrance component. Recent studies revealed that aromatherapy using essential oils may provide clinical benefits and could be used as an alternative medical treatment for hypertension $[1,2,5,6]$, hypotension $[1,3]$, cognitive

\footnotetext{
*Correspondence: okazaki@sports.osaka-cu.ac.jp

${ }^{1}$ Department of Environmental Physiology for Exercise, Osaka City

University Graduate School of Medicine, 3-3-138 Sugimoto Sumiyoshi, Osaka 558-8585, Japan

Full list of author information is available at the end of the article
}

dysfunction [4], and physical and psychological stress and exhaustion [2, 7-11]. For example, Fernandez et al. [3] reported that the anti-hypotensive effect of exposure to $1 \mathrm{~mL}$ of rosemary essential oil every $8 \mathrm{~h}$ was maintained in hypotensive patients when compared to the pretreatment period. In another study, Goepfert et al. [1] demonstrated that the systolic and diastolic blood pressure (BP) and heart rate (HR) of patients in palliative care were decreased after exposure to lavender essential oil for $10 \mathrm{~min}$ when compared to a placebo trial.

As for the physiological effects of aromatherapy, the responses of cardiovascular variables, including BP and HR, to fragrance inhalation of essential oils have been

c) The Author(s) 2020. This article is licensed under a Creative Commons Attribution 4.0 International License, which permits use, sharing, adaptation, distribution and reproduction in any medium or format, as long as you give appropriate credit to the original author(s) and the source, provide a link to the Creative Commons licence, and indicate if changes were made. The images or other third party material in this article are included in the article's Creative Commons licence, unless indicated otherwise in a credit line to the material. If material is not included in the article's Creative Commons licence and your intended use is not permitted by statutory regulation or exceeds the permitted use, you will need to obtain permission directly from the copyright holder. To view a copy of this licence, visit http://creativeco mmons.org/licenses/by/4.0/. 
investigated [1-3, 5, 6, 12-16]. In experimental animals, it has been reported that olfactory stimulation with the scent of grapefruit essential oil elevates mean BP [13, 14, $16,17]$, whereas olfactory stimulation with the scent of lavender essential oil decreases mean BP $[15,16]$. Similarly, in humans, Sayorwan et al. [5] demonstrated that systolic and diastolic BP and HR decreased with fragrance inhalation of lavender essential oil compared with the control condition. We also reported that mean BP and HR decreased with fragrance inhalation of sweet marjoram essential oil when compared to the control condition [12].

The change in hemodynamics with fragrance inhalation of essential oils is reportedly associated with changes in the mechanisms responsible for the autonomic control of circulation. In experimental animals, Niijima et al. [18] reported that olfactory stimulation with grapefruit essential oil evoked a slight increase in the nerve activity of the sympathetic branch innervating the white adipose tissue of the epididymis. In addition, Tanida et al. [16] demonstrated that the elevation in mean BP with olfactory stimulation with grapefruit essential oil was observed alongside increased renal sympathetic nerve activity. In humans, one previous study using power spectral analysis of BP fluctuations in normal adults reported that fragrance inhalation of essential oils such as pepper, grapefruit, estragon, or fennel oil induced a 1.7to 2.5 -fold increase in the low-frequency component of systolic BP, indicating increased sympathetic nerve activity. On the other hand, fragrance inhalation of rose oil or patchouli oil resulted in an approximate $40 \%$ decrease in the index compared to the control, indicating decreased sympathetic nerve activity [19]. Moreover, in the study, fragrance inhalation of rose oil resulted in a $30 \%$ decrease in plasma adrenaline concentration [19]. However, no previous study has provided direct evidence showing the association between changes in sympathetic nerve activity and changes in hemodynamics with fragrance inhalation of essential oils in humans.

Sympathetic nerve activity can be measured directly in humans via microneurography [20-22]. This technique allows for direct measurement of electrical activity in postganglionic sympathetic nerves using a minimally invasive, approach in which tungsten electrodes are percutaneously inserted into peripheral nerves [20, 23, 24]. Therefore, the aim of this study was to evaluate the effects of fragrance inhalation of grapefruit essential oil, which has been reported to increase sympathetic nerve activity and BP in rats [25], on changes in hemodynamics and muscle sympathetic nerve activity (MSNA) in humans (study 1). We hypothesized that fragrance inhalation of grapefruit essential oil would induce an increase in BP, and that this increase would be associated with changes in MSNA. Additionally, to elucidate whether the observed effects of fragrance inhalation of grapefruit essential oil on hemodynamics and MSNA were induced through a stress response to the fragrance inhalation, we also evaluated plasma cortisol concentrations and the participants' subjective emotions related to the fragrance (study 2). We also determined plasma adrenocorticotropic hormone $(\mathrm{ACTH})$ concentrations in addition to cortisol and catecholamine to evaluate the effects of the fragrance inhalation on the activity of the hypothalamic-pituitary-adrenal axis and the sympathetic-adrenal-medullary axis (study 3 ). The results of this study will provide a better understanding of the mechanism of the changes in hemodynamics with fragrance inhalation of essential oils and aid us in developing effective strategies for the use of aromatherapy in clinical settings.

\section{Methods}

\section{Subjects}

In study 1 , thirteen healthy male volunteers participated. Their age, height and body weight were $21 \pm 2.1$ years, $173 \pm 5.6 \mathrm{~cm}$, and $69 \pm 8.3 \mathrm{~kg}$ [means \pm standard deviation (SD)], respectively. In study 2 , another nine healthy male volunteers participated. Their age, height, and body weight were $21 \pm 2.2$ years, $173 \pm 6.2 \mathrm{~cm}$, and $71 \pm 15 \mathrm{~kg}$, respectively. In study 3 , additional nine healthy male volunteers participated. Their age, height, and body weight were $23 \pm 2.8$ years, $172 \pm 4.2 \mathrm{~cm}$, and $76 \pm 22 \mathrm{~kg}$, respectively. Exclusion criteria for the recruitment of subjects in both studies were: those diagnosed with cardiovascular, hypertension, respiratory, metabolic, or endocrine disease; and those who smoked or were taking prescribed medication.

\section{Fragrance inhalation}

To examine the effects of fragrance inhalation of grapefruit essential oil on MSNA, hemodynamics, respiratory variables, autonomic control of circulation, and stress hormone, all subjects in studies 1 and 2 were tested while inhaling plain air (baseline) and while inhaling the fragrance of grapefruit essential oil. In study 3 , subjects were tested at baseline and while inhaling air containing no fragrance or air containing fragrance in random order to exclude any effects of the circadian rhythm on the hormonal variables. We used a quantitative and accurate technique developed in our previous study to administer the fragrance [12]. Briefly, undiluted grapefruit essential oil (Citrus paradise oil; Seikatsunoki, Tokyo, Japan) was diffused at a rate of $0.27 \mathrm{~mL} /$ min using an ultrasonic aroma diffuser (DOSHISHA DAM-1101, Doshisha Corporation, Osaka, Japan) into an acrylic box $(60 \mathrm{~cm} \times 60 \mathrm{~cm} \times 60 \mathrm{~cm})$ with two drain hoses. Flow air was injected at a constant speed (30 L/ 
min) into the box via a drain hose and controlled using a gas regulator. Thus, the essential oil diffused in the box was diluted with injected air at a given concentration $\left(0.9 \times 10^{-2} \mathrm{~mL} / \mathrm{L}\right)$. The air containing fragrance was collected into a Douglas bag $(200 \mathrm{~L})$ attached to the other drain hose. Subjects wore a face mask with one-way valves throughout experiment and inhaled either air containing no fragrance or air containing fragrance from the Douglas bag. We used a three-way stopcock with balloon valves to switch the lines. Exhaled gases were collected through a hose attached to another Douglas bag to avoid diffusion of the fragrance into the room.

\section{Protocol}

In studies 1 and 2, the experiment was performed in the midmorning (study $1, n=8$; study $2, n=6$ ) or the afternoon (study $1, n=5$; study $2, n=3$ ). In study 3 , all experiments were performed in the afternoon to evening. Subjects were instructed to abstain from consuming caffeinated or alcoholic beverages and to refrain from vigorous physical activity for at least $24 \mathrm{~h}$ before the experiment. Subjects arrived at the laboratory having fasted for at least $2 \mathrm{~h}$ after a light meal. The experiment was performed in quiet, environmentally controlled laboratories with an ambient temperature of $\sim 28.0{ }^{\circ} \mathrm{C}$ with the subjects in a supine position. The concentration of essential oil component in the air and the duration of inhalation were determined from results of pilot studies.

\section{Study 1}

After instrumentation, and at least 10 min after a satisfactory nerve recording site had been determined, the experiment was started. While the subjects remained resting in a supine position and breathing through the facemask, 5-min baseline inhalation and 10-min fragrance inhalation were completed in random and counter-balanced orders. The fragrance inhalation period was followed by a 10-min recovery period. MSNA, hemodynamics, and respiratory variables were continuously recorded.

\section{Study 2}

After instrumentation, subjects were placed in the supine position and an intravenous catheter was inserted into an antecubital vein of the left arm for blood samples. At least $20 \mathrm{~min}$ after the insertion, the experiment was started. In the same manner as in study 1,5-min baseline inhalation and 10-min fragrance inhalation were completed. Blood samples were taken after 5 min of baseline inhalation and after 5 and $10 \mathrm{~min}$ of fragrance inhalation.

\section{Study 3}

All of the procedures before the start of test were same as in study 2. Subjects underwent the control trial and the fragrance trial in random order. After 10-min baseline, subjects inhaled air containing no fragrance (control trial) or air containing fragrance (fragrance trial) for $10 \mathrm{~min}$. At least $20 \mathrm{~min}$ for recovery was inserted between trials. Blood samples were taken after $10 \mathrm{~min}$ of baseline and at 5 and $10 \mathrm{~min}$ after the start of inhalation.

\section{Measurements \\ Study 1 \\ Muscle sympathetic nerve activity}

MSNA signals were obtained using microneurography $[20,26,27]$. Briefly, a recording electrode was placed in the left peroneal nerve fascicles at the popliteal fossa. A reference $\mathrm{Ag}-\mathrm{AgCl}$ electrode was placed at the skin surface $2-3 \mathrm{~cm}$ apart from the recording electrode. The nerve signals were amplified (gain 100,000), band-pass filtered $(0.7-3 \mathrm{kHz})$, full-wave rectified, and integrated by a capacitance integrated circuit with a time constant of $0.1 \mathrm{~s}$ to obtain a mean voltage neurogram using isolated amplifiers (MEG-1251, Nihon Kohden, Tokyo, Japan) and an integrator (E1-601G, Nihon Kohden, Tokyo, Japan). Criteria for adequate MSNA recording without any skin sympathetic nerve signals included (1) pulse synchrony; (2) facilitation during the hypotensive phase of the Valsalva maneuver, and suppression during the hypertensive overshoot phase after release; (3) increases in response to breath holding; and (4) insensitivity to emotional stimuli, deep breath, or gentle skin touch within the innervated area [26]. Before starting the experiment, we waited for at least 10 min after we observed stable data on HR, BP, and MSNA signals, to avoid any effect of the maneuvers of sympathetic stimulation for checking MSNA signals on the measurements.

\section{Hemodynamics, respiratory variables, and subjective emotion related to the fragrance}

$\mathrm{R}-\mathrm{R}$ intervals (RRI) and HR were obtained from lead II of the electrocardiogram tracings (BSM-7201; Nihon Kohden Co., Tokyo, Japan) and beat-by-beat blood pressure (BP) was recorded noninvasively using finger photoplethysmography (Finometer MIDI; Finapres Medical System, Amsterdam, the Netherlands). Mean BP was calculated as [systolic BP (SBP) - diastolic BP (DBP)]/3+DBP. Respiratory variables were determined from oxygen and carbon dioxide fractions in the expired gas and the ventilatory volume (AE-310 s, Minato, Osaka, Japan).

Immediately after each experimental trial, the subjects were asked to rate their valence $(0$, unpleasant; to 9 , 
pleasant) and arousal (0, relaxing; to 9, stimulating) [28] to allow us to evaluate their subjective emotion related to the fragrance using a 10-point scale.

\section{Study 2 \\ Hemodynamics, respiratory variables, and subjective emotion related to the fragrance}

HR was obtained as in study 1. Beat-by-beat BP was recorded noninvasively by tonometry (BP-608 Evolution II, Omron-Colin, Tokyo, Japan). Respiratory variables were determined from oxygen and carbon dioxide fractions in the expired gas and the ventilatory volume (ARCO2000-MET, Arcosystem, Chiba, Japan). Subjective emotions related to the fragrance were obtained as in study 1 .

\section{Blood constituents}

Blood samples were transferred to a vacuum blood-sample tube containing $1.5 \mathrm{mg} / \mathrm{mL}$ EDTA-2Na and centrifuged at $5{ }^{\circ} \mathrm{C}$ for $15 \mathrm{~min}$. The separated plasma sample was stored at $-80{ }^{\circ} \mathrm{C}$ operating point until used to measure the plasma concentrations of cortisol (chemiluminescent immunoassay, LSI, Tokyo, Japan) and noradrenaline (high-performance liquid chromatography, LSI).

\section{Study 3}

\section{Blood constituents}

The procedures for blood constituents were same as in study 2 . The plasma concentrations of cortisol and $\mathrm{ACTH}$ (chemiluminescent immunoassay, SRL, Tokyo, Japan), adrenaline and noradrenaline (high-performance liquid chromatography, SRL) were determined.

\section{Data analysis}

Data were stored on a computer (500 $\mathrm{Hz}$ sampling rate) using a computer-based data acquisition and analysis system (Powerlab 16SP and LabChart 7; ADInstruments, Sydney, Australia). Beat-by-beat HR, RRI, SBP, and DBP were extracted from the obtained data, and MSNA bursts were identified from the integrated neurogram using a MATLAB program (R2018b, The MathWorks, Natick, MA) with a 3:1 signal-to-noise ratio threshold within a $0.5 \mathrm{~s}$ search window and an expected burst reflex latency of $1.2 \mathrm{~s}$ from the preceding $\mathrm{R}$ waves $[29,30]$. MSNA bursts were confirmed by an experienced microneurographer. Quantitative indices of MSNA were the number of bursts per minute (burst frequency, bursts/min), the number of bursts per 100 heart beats (burst incidence, bursts/100 beats), and total activity (total MSNA, units).

Sympathetic and cardiovagal baroreflex sensitivity (BRS) were calculated using data obtained during the last 2 min of baseline and fragrance inhalation. Sympathetic BRS was assessed using the slope of the linear correlation between total MSNA or MSNA burst incidence and DBP calculated over a 3-mmHg bin during spontaneous breathing after statistical weighting [31]. Cardiovagal BRS was also assessed using the slope of the linear correlation between changes in RRI or HR and changes in SBP [32]. The SD of RRI, HR, SBP, and DBP were also calculated $[29,33]$.

\section{Statistical analysis}

All data were analyzed using a statistical software (SigmaPlot 14.0, Systat Software, Inc., San Jose, USA). In study 1 , the minute average was calculated for each variable. Fragrance inhalation was separated into two phases: the first and last $5 \mathrm{~min}$. The two phases were compared with the 5-min baseline value. Two-way analysis of variance (ANOVA) with repeated-measures (trial, BL vs. inhalation; time) was used to test the effects of fragrance inhalation on each variable. Subsequent post hoc tests to determine significant differences among various pairwise comparisons were performed using Fisher's least significant difference test. The changes in DBP $(\triangle \mathrm{DBP})$ and MSNA burst frequency ( $\Delta$ burst frequency) with fragrance inhalation were calculated by subtracting the average value of 5-min baseline from the average value of the last 5-min of inhalation. Pearson's product-moment correlation coefficient was used to evaluate the relationships between $\triangle \mathrm{DBP}$ and $\triangle$ burst frequency or the delta values and the baseline values. Unpaired t-test was used to determine significant difference in $\triangle \mathrm{DBP}$ between the groups of subjects in the midmorning and in the afternoon. In study 2, the 5-min average was calculated for hemodynamics and respiratory variables. One-way ANOVA with repeated-measures (BL vs. inhalation) was used to test the effects of fragrance inhalation on each variable. In study 3, two-way ANOVA with repeatedmeasures (trial, BL vs. inhalation; time) was used to test the effects of fragrance inhalation on each variable. Subsequent post hoc tests to determine significant differences among various pairwise comparisons were performed using Duncan's test. Values are expressed as the means \pm SDs except as noted. $P<0.05$ was considered statistically significant.

\section{Results}

Study 1

Figure 1 shows the hemodynamic responses to fragrance inhalation of grapefruit essential oil. HR remained unchanged, whereas BPs tended to increase during fragrance inhalation. We found a significant interaction effect (trial $\times$ time, $P=0.035$ ) on $\mathrm{DBP}$, which showed a significant increase at 9 to $10 \mathrm{~min}$ of fragrance inhalation when compared to the baseline. 

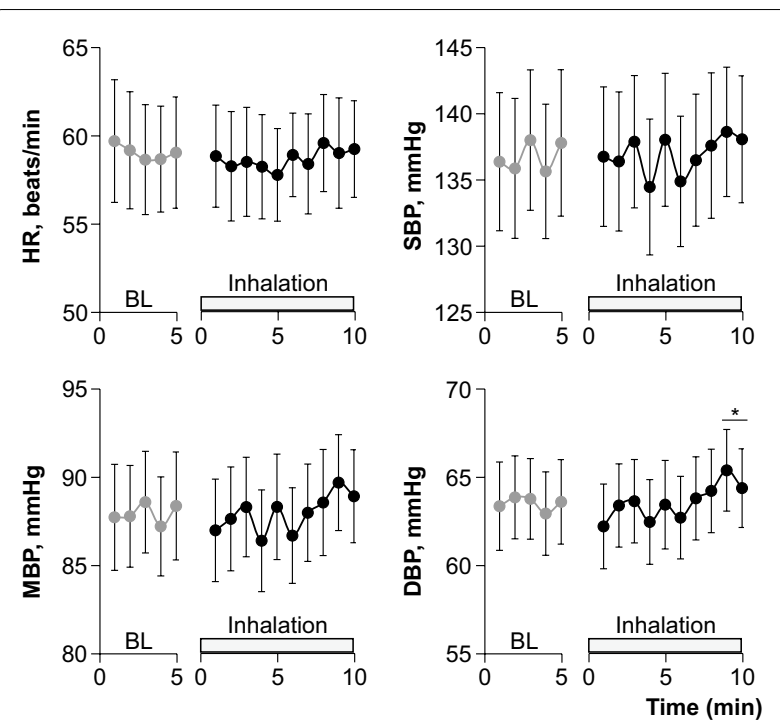

Fig. 1 Hemodynamic responses to fragrance inhalation of grapefruit essential oil in study 1. BL baseline, HR heart rate, SBP systolic blood pressure, MBP mean blood pressure, DBP diastolic blood pressure. Values are expressed as the means \pm standard errors. ${ }^{*} P<0.05$ vs. BL

Table 1 Respiratory variables during baseline and fragrance inhalation of grapefruit essential oil in study 1

\begin{tabular}{lccc}
\hline & \multicolumn{1}{c}{ BL } & Inh-5 min & Inh-10 min \\
\hline$V_{E^{\prime}} \mathrm{L} / \mathrm{min}$ & $8.4 \pm 2.9$ & $8.5 \pm 2.8$ & $8.1 \pm 3.3$ \\
$\mathrm{RR}, \mathrm{breaths} / \mathrm{min}$ & $16.2 \pm 5.2$ & $16.2 \pm 4.9$ & $16.1 \pm 4.8$ \\
$V_{\mathrm{O} 2}, \mathrm{~mL} / \mathrm{min}$ & $236 \pm 91$ & $239 \pm 93$ & $230 \pm 96$ \\
$V_{\mathrm{CO} 2}, \mathrm{~mL} / \mathrm{min}$ & $196 \pm 80$ & $207 \pm 89$ & $194 \pm 89$ \\
RER & $0.83 \pm 0.08$ & $0.86 \pm 0.11$ & $0.84 \pm 0.12$
\end{tabular}

Values are means $\pm S D$. Average data for 5 min of baseline $(B L)$ and for the first (Inh-5 min) and last half (Inh-10 min) of fragrance inhalation are shown

$V_{E^{\prime}}$ minute ventilation; $R R$, respiratory rate, $V_{\mathrm{O} 2}$, oxygen consumption rate; $V_{\mathrm{CO} 2}$, carbon dioxide production rate; $R E R$, respiratory exchange ratio

As shown in Table 1, respiratory variables remained unchanged during fragrance inhalation.

Figure 2 shows original recordings of the integrated MSNA from one representative subject during baseline and fragrance inhalation. Responses of MSNA variables to fragrance inhalation of grapefruit essential oil are shown in Fig. 3. We did not find any significant change in burst incidence, burst frequency, or total MSNA with fragrance inhalation. Nonetheless, as shown in Fig. 4, we found a significant linear correlation $(R=0.74, P=0.006)$ between $\Delta$ burst frequency and $\triangle \mathrm{DBP}$, indicating that the changes in DBP with fragrance inhalation were associated with changes in MSNA. Importantly, a partial correlation coefficient was significant even when MSNA burst frequency $(R=0.82, P<0.001)$ or $\mathrm{DBP}(R=0.72, P=0.006)$ at baseline was included as a variable. Furthermore, there were no significant correlations between MSNA burst frequency at baseline and $\Delta$ burst frequency $(R=-0.14$, $P=0.65)$ or $\mathrm{DBP}$ at baseline and $\triangle \mathrm{DBP}(R=0.24$, $P=0.42)$. Intriguingly, $\triangle \mathrm{DBP}$ showed a significant negative linear correlation with MSNA burst frequency at baseline $(R=-0.58, P=0.037)$, while there were no significant correlations between DBP and MSNA variables at baseline $(P>0.50) . \triangle \mathrm{DBP}$ was not significantly different between the groups of subjects in the midmorning and in the afternoon $(P=0.84)$.

Sympathetic and cardiovagal BRS are summarized in Table 2. Sympathetic BRS calculated between total MSNA or MSNA burst incidence and DBP and cardiovagal BRS calculated between RRI or HR and SBP remained unchanged with fragrance inhalation. We confirmed that the relationships between total MSNA and DBP $\left(R^{2}\right.$ value; BL, $0.52 \pm 0.35$; inhalation, $0.57 \pm 0.33)$, MSNA burst incidence and DBP ( $R^{2}$ value; BL, $0.54 \pm 0.29$; inhalation, $0.61 \pm 0.32)$, RRI and SBP $\left(R^{2}\right.$ value; $\mathrm{BL}, 0.41 \pm 0.15$; inhalation, $0.41 \pm 0.15)$, and $\mathrm{HR}$ and $\mathrm{SBP}\left(R^{2}\right.$ value; $\mathrm{BL}$, $0.41 \pm 0.16$; inhalation, $0.42 \pm 0.15$ ) were all significant in each subject. There were no significant effects of inhalation on the SDs of RRI (BL, 71.5 $\pm 33.4 \mathrm{~ms}$; inhalation, $66.1 \pm 34.7 \mathrm{~ms} ; P=0.512)$, HR (BL, $4.2 \pm 2.1 \mathrm{bpm}$; inhalation, $4.1 \pm 2.0 \mathrm{bpm}$; $\mathrm{P}=0.908)$, SBP $(\mathrm{BL}, 7.8 \pm 3.9 \mathrm{mmHg}$; inhalation, $6.4 \pm 1.9 \mathrm{mmHg} ; P=0.126)$, or DBP (BL, $4.1 \pm 1.5 \mathrm{mmHg}$; inhalation, $3.9 \pm 1.0 \mathrm{mmHg} ; P=0.599)$.

Figure 5 shows a bi-dimensional representation of the arousal and valence ratings of fragrance inhalation of grapefruit essential oil in study 1 . The evaluation of subjective emotion related to the fragrance of grapefruit essential oil revealed a majority of ratings on the pleasant (valence; $6.3 \pm 0.9$ ) and relaxing parts of the scale (arousal; $3.1 \pm 1.7$ ). Similar results were obtained in study 2 (valence; $6.7 \pm 1.9$, arousal; $4.1 \pm 2.6$ ).

\section{Study 2}

Similar to study 1 and as shown in Table 3, HR remained unchanged and DBP increased significantly during the last $5 \mathrm{~min}$ of fragrance inhalation when compared to baseline, respiratory variables remained unchanged during fragrance inhalation. The plasma noradrenaline level remained unchanged, whereas the plasma cortisol level decreased significantly during the last $5 \mathrm{~min}$ of fragrance inhalation when compared to baseline (Fig. 6).

\section{Study 3}

As shown in Fig. 7, there is no significant effect of trial on the plasm adrenaline and noradrenaline levels, and those remained unchanged during fragrance inhalation in both 

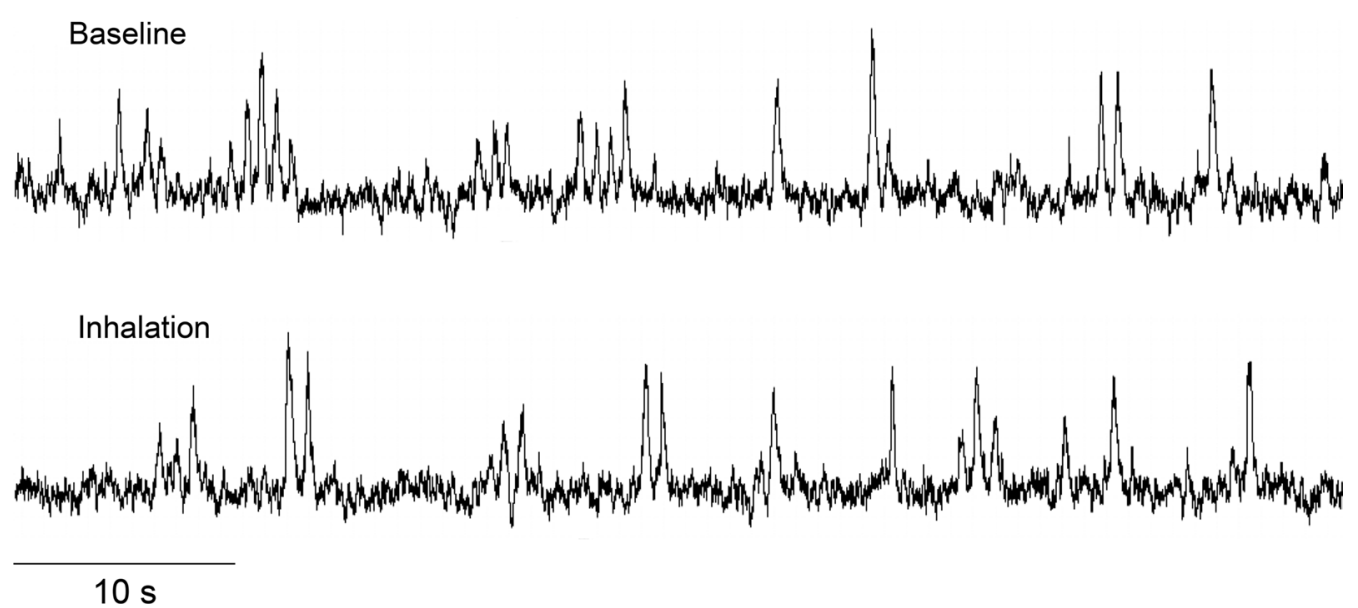

Fig. 2 Original recordings of the integrated muscle sympathetic nerve activity from one representative subject in study 1
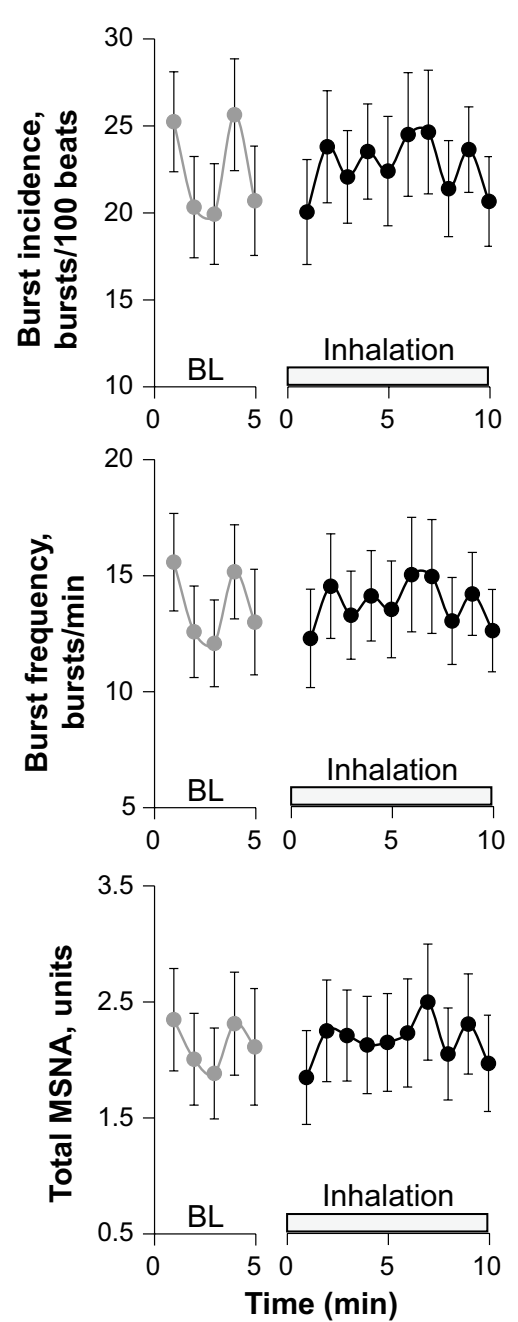

Fig. 3 Responses of muscle sympathetic nerve activity (MSNA) variables to fragrance inhalation of grapefruit essential oil in study 1. $B L$ baseline. Values are expressed as the means \pm standard errors

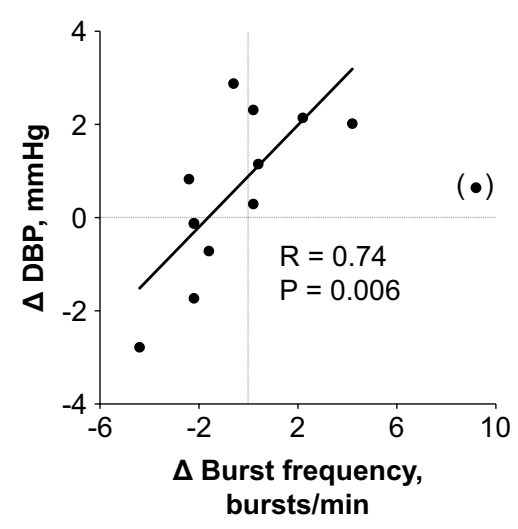

Fig. 4 Relationship between changes in diastolic blood pressure $(\triangle \mathrm{DBP})$ with fragrance inhalation of grapefruit essential oil from baseline and those in burst frequency of MSNA ( $\triangle$ burst frequency). Values are calculated as [5 min average for last half of fragrance inhalation] - [5 min average for baseline] for each individual. Data from one subject (shown in parentheses) was excluded from the analysis as an outlier in the Grubbs test

Table 2 Sympathetic and cardiovagal baroreflex sensitivity during baseline and fragrance inhalation of grapefruit essential oil in study 1

BL Inhalation

Sympathetic baroreflex sensitivity

$\begin{array}{lcr}\text { DBP-total MSNA, units/mmHg } & -0.66 \pm 0.73 & -0.86 \pm 0.82 \\ \begin{array}{c}\text { DBP-burst incidence, bursts.100 } \\ \text { beats }{ }^{-1} \cdot \mathrm{mmHg}^{-1}\end{array} & -1.3 \pm 1.8 & -2.0 \pm 1.9 \\ \begin{array}{l}\text { Cardiovagal baroreflex sensitivity } \\ \text { SBP-RRI, } \mathrm{ms} / \mathrm{mmHg}\end{array} & 10.8 \pm 5.6 & 11.9 \pm 9.6 \\ \text { SBP-HR, bpm } / \mathrm{mmHg} & -0.60 \pm 0.22 & -0.66 \pm 0.32\end{array}$

Values are means $\pm S D$. DBP diastolic blood pressure, MSNA muscle sympathetic nerve activity, $S B P$ systolic blood pressure, $R R I \mathrm{R}-\mathrm{R}$ interval, $H R$ heart rate. $B L$ baseline; Inhalation, fragrance inhalation of grapefruit essential oil 


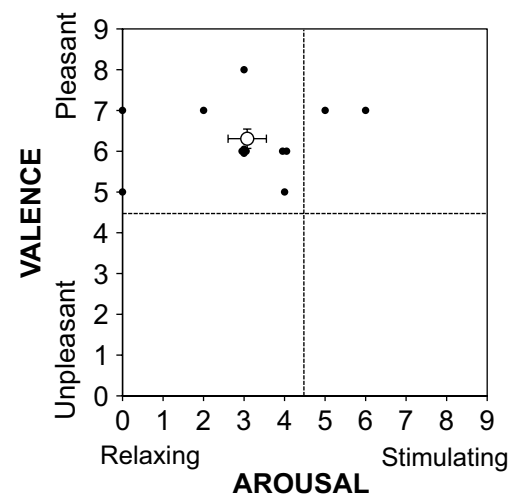

Fig. 5 Bi-dimensional representation of the arousal and valence ratings of fragrance inhalation of grapefruit essential oil in study 1. The open circle indicates the mean \pm standard errors, and the closed circles indicate the individual value for each subject

Table 3 Hemodynamics and respiratory variables during baseline and fragrance inhalation of grapefruit essential oil in study 2

\begin{tabular}{lccc}
\hline & BL & Inh-5 min & Inh-10 min \\
\hline HR, beats/min & $54.3 \pm 7.4$ & $53.8 \pm 6.8$ & $53.7 \pm 7.0$ \\
SBP, mmHg & $124 \pm 15$ & $126 \pm 15$ & $128 \pm 3$ \\
MBP, mmHg & $87 \pm 13$ & $89 \pm 13$ & $90 \pm 12$ \\
DBP, mmHg & $68 \pm 12$ & $70 \pm 13$ & $71 \pm 12^{*}$ \\
$V_{E}, \mathrm{~L} / \mathrm{min}$ & $8.5 \pm 4.9$ & $8.3 \pm 4.6$ & $8.3 \pm 4.8$ \\
$\mathrm{RR}, \mathrm{breaths} / \mathrm{min}$ & $14.2 \pm 6.4$ & $12.7 \pm 6.7$ & $13.4 \pm 4.2$ \\
$\mathrm{~V}_{\mathrm{O} 2}, \mathrm{~mL} / \mathrm{min}$ & $285 \pm 176$ & $302 \pm 175$ & $290 \pm 176$ \\
$\mathrm{~V}_{\mathrm{CO} 2}, \mathrm{~mL} / \mathrm{min}$ & $220 \pm 136$ & $227 \pm 131$ & $227 \pm 139$ \\
$\mathrm{RER}$ & $0.78 \pm 0.06$ & $0.76 \pm 0.06$ & $0.78 \pm 0.06$ \\
\hline
\end{tabular}

Values are means $\pm S D$. Average data for $5 \mathrm{~min}$ of baseline $(\mathrm{BL})$ and for the first (Inh-5 min) and last half (Inh-10 min) of fragrance inhalation are shown. ${ }^{*} P<0.05$ vs. BL

$H R$ heart rate, $S B P$ systolic blood pressure, $M B P$ mean blood pressure, $D B P$ diastolic blood pressure, $V_{\mathrm{E}}$ minute ventilation, $R R$ respiratory rate, $V_{O 2}$ oxygen consumption rate, $V_{\mathrm{CO} 2}$ carbon dioxide production rate, $R E R$ respiratory exchange ratio

trials. Importantly, the plasma cortisol and ACTH levels decreased significantly at 10 min of fragrance inhalation when compared to baseline in the fragrance trial while those remained unchanged in the control trial, although there are no significant effect of trial on these variables.

\section{Discussion}

The main findings of this study are as follows: (1) DBP increased during fragrance inhalation of grapefruit essential oil while other variables remained unchanged; (2) there was a significant linear correlation between changes in DBP with fragrance inhalation and changes in MSNA burst frequency; and (3) the plasma cortisol concentration decreased with fragrance inhalation. These results suggest, for the first time, that changes in BP with fragrance inhalation of an essential oil are associated with changes in MSNA even with decreased stress hormone.

We showed that fragrance inhalation of grapefruit essential oil induced a significant increase in DBP without an increase in HR while subjects rested in the supine position. Importantly, we successfully reproduced the observations in two different studies with the same protocol (Fig. 1 and Table 3) by using a quantitative and accurate technique to apply a given fragrance [12]. Our results extended to humans previous observations on experimental animals that olfactory stimulation with the fragrance of grapefruit essential oil elevated mean BP without an increase in HR [13, 14, 16, 17].

Most importantly, for the purpose of the present study, we found that changes in DBP with fragrance inhalation were correlated with changes in MSNA burst frequency (Fig. 4). We confirmed that the changes in DBP and MSNA burst frequency with fragrance inhalation were not associated merely with baseline values. Previous studies have reported that olfactory stimulation in rats with the fragrance of grapefruit oil increased renal sympathetic nerve activity [16] or nerve activity of the sympathetic branch innervating the white and brown adipose tissues [13] and suppressed gastric vagal nerve activity $[13,18]$. The present study supported these observations and suggest that, in humans, fragrance inhalation of grapefruit essential oil increased BP through the mechanisms of change in MSNA. We supposed that the changes in BP were not large enough to observe a simultaneous increase in sympathetic nerve activity, which may explain why we failed to find significant increases in MSNA variables and plasma noradrenaline concentration with fragrance inhalation in the present study. Niijima et al. [18] showed that, in rats, an olfactory stimulation with grapefruit oil (diluted 1000 times) for 10 min evoked a significant increase in sympathetic nerve activity, but not with a thinner solution (1/10,000 in concentration). It is expected that MSNA variables and plasma noradrenaline concentration may increase with a greater increase in $\mathrm{BP}$ if we were to use a higher/optimal concentration of grapefruit essential oil.

As for the mechanisms involved in the increase in BP and sympathetic nerve activity with fragrance inhalation of grapefruit essential oil, previous studies on rats have suggested that olfactory stimulation with the fragrance of grapefruit oil affect autonomic neurotransmission to induce an increase in BP through central mechanisms $[14,16,34,35]$. Tanida et al. reported that olfactory stimulation with the fragrance of grapefruit $[16,34]$ oil or its active component limonene [14] similarly induced an elevation in renal sympathetic nerve activity and $\mathrm{BP}$ 

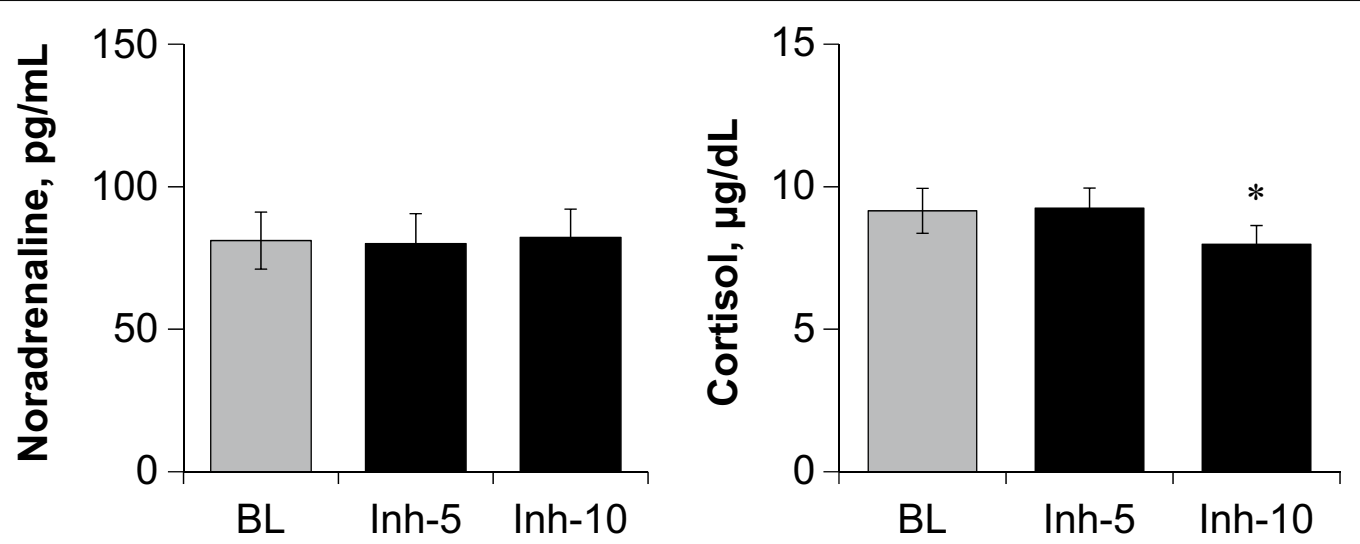

Fig. 6 Responses of plasma concentrations of noradrenaline and cortisol to fragrance inhalation of grapefruit essential oil in study 2 . Values are expressed as the means \pm standard errors. BL, baseline; Inh- 5 and Inh-10, at 5 and 10 min of fragrance inhalation, respectively. ${ }^{*}<<0.05$ vs. BL
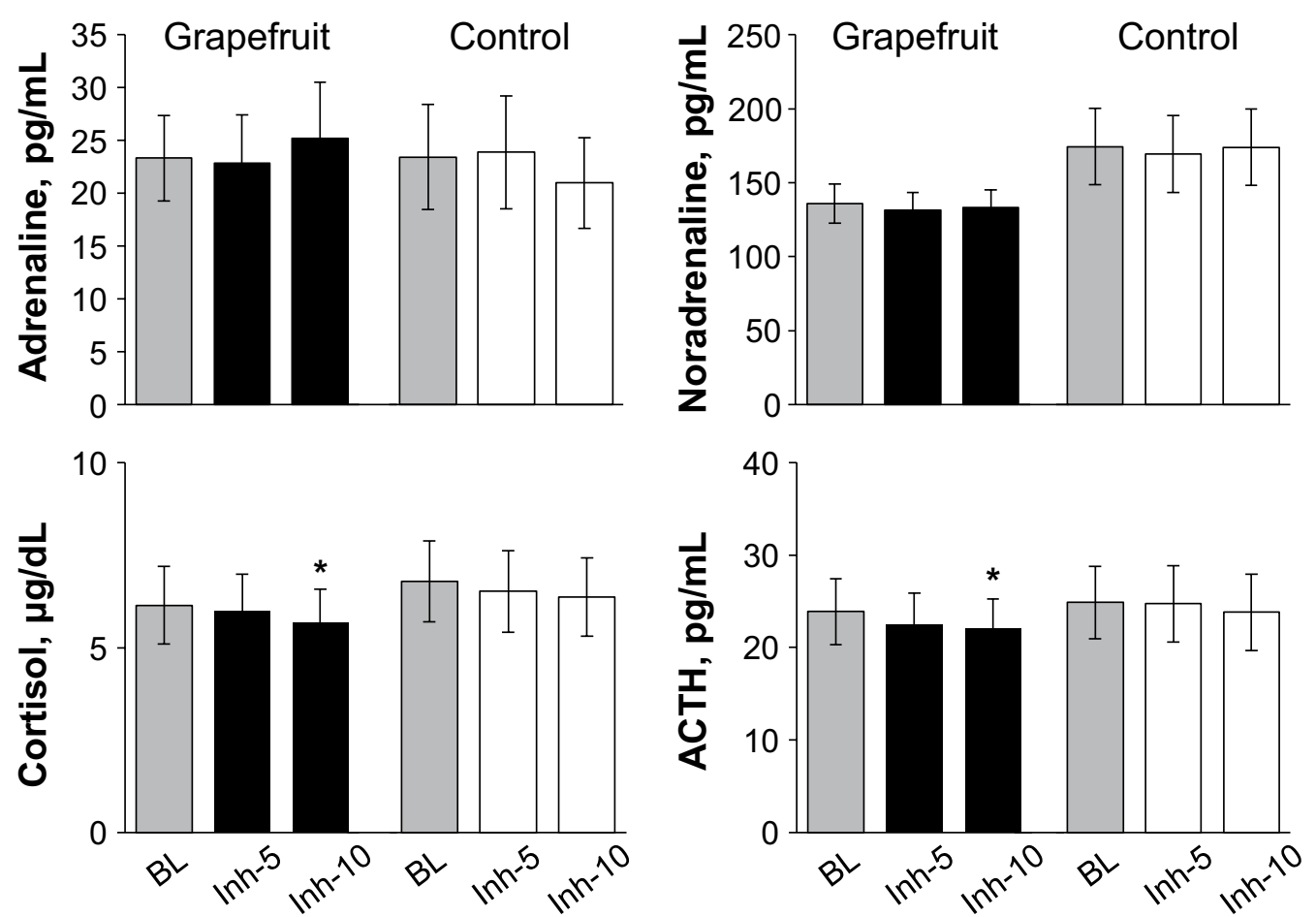

Fig. 7 Responses of plasma concentrations of cortisol, adrenocorticotropic hormone (ACTH), adrenaline, and noradrenaline during the control trial and the grapefruit fragrance trial in study 3 . Values are expressed as the means \pm standard errors. BL baseline; Inh-5 and Inh-10, at 5 and 10 min of fragrance inhalation, respectively. ${ }^{*} P<0.05$ vs. BL

and suppressed gastric vagal nerve activity; moreover, intracranial injection of diphenhydramine, a histamine H1-receptor antagonist, or bilateral electrolytic lesions of the hypothalamic suprachiasmatic nucleus (SCN) completely eliminated the autonomic and cardiovascular response to grapefruit [35] and limonene [14]. Intriguingly, the elevation in renal sympathetic nerve activity and BP with olfactory stimulation with the fragrance of grapefruit oil observed in wild-type mice was not observed in clock mutant mice [16] or Cry1 and Cry2 double knockout $[\mathrm{Cry}(-/-)]$ mice [34]. Indeed, it has been reported that limonene is observed within brain following inhalation in mouse [36] and that the intranasal delivery of the molecule to central nerves system 
via axonal or extracellular transport of the olfactory nerve takes at least 5-10 min after administration [37, 38]. Therefore, it is conceivable that the increase in DBP with the changes in MSNA induced by fragrance inhalation of grapefruit essential oil observed in the present study was provoked via the SCN activated by limonene through a pathway in the olfactory system and that the central histaminergic nervous system and the molecular clock mechanism in the SCN are involved in mediating these responses. In contrast, inhalation of odor molecule activates cell of the olfactory epithelium in the nasal cavity and thus induces olfactory perception and its effect through the olfactory nervous system [39]. However, considering that the onset of olfactory perception is very rapid and acclimatization soon take place [40, 41], this mechanism would not be involved, because, in the present study, the increase in DBP required 9-10 min after the onset of inhalation. Coupled with the observations that sympathetic and cardiovagal BRS remained unchanged (Table 2), the fragrance inhalation of grapefruit essential oil seems to activate the SCN to increase the operating point of BP regulation [42].

There are substantial individual variations in the responses of DBP and MSNA to fragrance inhalation of grapefruit essential oil. Intriguingly, the changes in DBP with fragrance inhalation were negatively correlated with MSNA burst frequency at baseline, indicating that the resting level of MSNA is a determinant of the individual variations, which may be associated with the effectiveness of aromatherapy. It is inferable that the response of the SCN to fragrance inhalation of grapefruit essential oil is associated with the baseline level of sympathetic tone. Hemodynamics and sympathetic regulation of $\mathrm{BP}$ at baseline and in response to pressor stimulus [43], as well as vasoconstriction in response to norepinephrine and $\beta$-adrenergic vasodilation, are known to be influenced by gender [44]. Our results are limited to men; therefore, inclusion of women may alter our findings. Further studies including women are required to elucidate the background mechanisms involved in the individual variations in cardiovascular and sympathetic responses to fragrance inhalation of grapefruit essential oil.

As far as we know, no previous study has reported the direct effects of grapefruit essential oil on blood vessels, though it has been known that olfactory receptor expression is observed in the aorta, renal, and iliac arteries and in the smooth muscle cells of small blood vessels in a variety of tissues including the heart, diaphragm, skeletal muscle, and skin [45]. It has been reported that intravenous injections of essential oil of Aniba rosaeodora induce a hypotensive response and that this response remained unchanged by pretreatment via bivagotomy [46]. Although we may not be able to exclude the effects of grapefruit essential oil on BP via local vasoconstrictive mechanisms; the involvement of these mechanisms would be low, as we observed the significant correlation between the changes in DBP and MSNA burst frequency with fragrance inhalation.

We observed that plasma cortisol level decreased with ACTH level during fragrance inhalation of grapefruit essential oil (Figs. 6 and 7), and determined the subjective emotions related to the fragrance to be pleasant and relaxing (Fig. 5). In contrast, plasma adrenaline and noradrenaline levels remained unchanged during fragrance inhalation of grapefruit essential oil (Figs. 6 and 7). These observations indicate that the increase in DBP and the changes in MSNA induced by fragrance inhalation of grapefruit essential oil were not induced through a stress response which enhances both of the sympathetic-adrenal-medullary axis and the hypothalamicpituitary-adrenal axis [47]. Recently, Takagi et al. [48] reported that fragrance inhalation of grapefruit essential oil recovers the reduction in the salivary level of secretory immunoglobulin A by mental stress in humans, indicating that the inhalation of grapefruit essential oil induced stress free actions. In addition, previous studies suggested that fragrance administration of oils though other than grapefruit essential oil attenuated an increase in salivary cortisol concentration to mental stress in humans $[49,50]$ or plasma ACTH level to physical stress in rats, while decreased the stress-induced activity of prefrontal cortex which regulates the activity of the hypothalamic-pituitary-adrenal axis in humans [51]. Indeed, we confirmed that plasma ACTH level decreased with cortisol level with the grapefruit fragrance inhalation (Fig. 7). These observations would indicate that the inhalation of grapefruit essential oil has a mechanism to decrease the activity of the hypothalamic-pituitary-adrenal axis. Importantly, cortisol has effects in the control of vascular smooth muscle tone by its permissive effects in potentiating vasoactive responses to catecholamines [52] and therefore might be associated with the cardiovascular changes with the fragrance inhalation. However, based on our observations that DBP increased while plasma cortisol level decreased with the grapefruit fragrance inhalation, we assumed that the increased DBP was not associated with the changes in cortisol but associated with the changes in MSNA.

\section{Limitations}

We did not determine the effects of fragrance inhalation of grapefruit essential oil on renin-angiotensin system and vasopressin levels which would be a possible mechanism of the observed changes in DBP via vasoconstriction. As far as we know, there is no previous study reporting the effects of fragrance inhalation of grapefruit essential oil on these 
mechanisms. Further studies are required to assess the involvement of these mechanisms on the cardiovascular changes after the grapefruit fragrance inhalation.

\section{Conclusion}

In conclusion, the fragrance inhalation of grapefruit essential oil induced an increase in DBP in healthy men. The changes in DBP with fragrance inhalation were correlated with changes in MSNA, even with decreased plasma cortisol concentrations. These results suggest, for the first time in humans, that the changes in BP with fragrance inhalation of an essential oil are associated with changes in MSNA. The activation of sympathetic nerve activity with fragrance inhalation without an increase in stress hormone may be one of the mechanisms involved in the positive effects and refreshment of aromatherapy in humans.

\section{Acknowledgements}

We are very grateful to the volunteers who participated in this study.

\section{Authors' contributions}

EK, SU, TM and KO conceived and designed the study. EK, DI, RT, AO, EM, SU, HN, TM and KO performed experiments. EK, YS, SU, TM and KO analyzed data. EK, RT, DI, HY, TM and KO edited and revised manuscript. All authors read and approved final manuscript.

\section{Funding}

This study was supported in part by a grant-in-aid for JSPS Research Fellows, JSPS KAKENHI Grant Number 18J15391 (to E. Kawai) from the Japan Society for the Promotion of Science.

\section{Availability of data and materials \\ Not applicable.}

\section{Ethics approval and consent to participate}

This study procedure was approved by the institutional review boards of our institution, and conformed to the standards set by the Declaration of Helsinki. The written informed consent from all subjects was obtained before participation in this study.

\section{Consent for publication}

Not applicable.

\section{Competing interests}

The authors declare that they have no competing interests.

\section{Author details}

${ }^{1}$ Department of Environmental Physiology for Exercise, Osaka City University Graduate School of Medicine, 3-3-138 Sugimoto Sumiyoshi, Osaka 558-8585, Japan. ${ }^{2}$ Research Center for Urban Health and Sports, Osaka City University, Osaka, Japan. ${ }^{3}$ Department of Physical Education, Faculty of Education, Gifu University, Gifu, Japan. ${ }^{4}$ Graduate School of Health Sciences, Morinomiya University of Medical Sciences, Osaka, Japan. ${ }^{5}$ Department of Sport and Health Sciences, Faculty of Sport and Health Sciences, Osaka Sangyo University, Daito, Osaka, Japan.

Received: 14 October 2019 Accepted: 30 December 2019

Published online: 30 January 2020

\section{References}

1. Goepfert M, Liebl P, Herth N, Ciarlo G, Buentzel J, Huebner J (2017) Aroma oil therapy in palliative care: a pilot study with physiological parameters in conscious as well as unconscious patients. J Cancer Res Clin Oncol 143:2123-2129. https://doi.org/10.1007/s00432-017-2460-0

2. Seol GH, Lee YH, Kang P, You JH, Park M, Min SS (2013) Randomized controlled trial for Salvia sclarea or Lavandula angustifolia: differential effects on blood pressure in female patients with urinary incontinence undergoing urodynamic examination. J Altern Complement Med 19:664-670. https://doi.org/10.1089/acm.2012.0148

3. Fernandez LF, Palomino OM, Frutos G (2014) Effectiveness of Rosmarinus officinalis essential oil as antihypotensive agent in primary hypotensive patients and its influence on health-related quality of life. J Ethnopharmacol 151:509-516. https://doi.org/10.1016/j.jep.2013.11.006

4. Jimbo D, Kimura Y, Taniguchi M, Inoue M, Urakami K (2009) Effect of aromatherapy on patients with Alzheimer's disease. Psychogeriatrics 9:173-179. https://doi.org/10.1111/j.1479-8301.2009.00299.x

5. Sayorwan W, Siripornpanich V, Piriyapunyaporn T, Hongratanaworakit T, Kotchabhakdi N, Ruangrungsi N (2012) The effects of lavender oil inhalation on emotional states, autonomic nervous system, and brain electrical activity. J Med Assoc Thai 95:598-606

6. Dong S, Jacob TJ (2016) Combined non-adaptive light and smell stimuli lowered blood pressure, reduced heart rate and reduced negative affect. Physiol Behav 156:94-105. https://doi.org/10.1016/j.physbeh.2016.01.013

7. Matsumoto T, Asakura H, Hayashi T (2014) Effects of olfactory stimulation from the fragrance of the Japanese citrus fruit yuzu (Citrus junos Sieb. ex Tanaka) on mood states and salivary chromogranin A as an endocrinologic stress marker. J Altern Complement Med 20:500-506. https://doi. org/10.1089/acm.2013.0425

8. Field T, Diego M, Hernandez-Reif M, Cisneros W, Feijo L, Vera Y, Gil K, Grina D, Claire He Q (2005) Lavender fragrance cleansing gel effects on relaxation. Int J Neurosci 115:207-222. https://doi.org/10.1080/0020745059 0519175

9. Diego MA, Jones NA, Field T, Hernandez-Reif M, Schanberg S, Kuhn C, McAdam V, Galamaga R, Galamaga M (1998) Aromatherapy positively affects mood, EEG patterns of alertness and math computations. Int J Neurosci 96:217-224

10. Watanabe E, Kuchta K, Kimura M, Rauwald HW, Kamei T, Imanishi J (2015) Effects of bergamot (Citrus bergamia (Risso) Wright \& Arn.) essential oil aromatherapy on mood states, parasympathetic nervous system activity, and salivary cortisol levels in 41 healthy females. Forsch Komplementmed 22:43-49. https://doi.org/10.1159/000380989

11. Matsubara E, Tsunetsugu Y, Ohira T, Sugiyama M (2017) Essential oil of Japanese cedar (Cryptomeria japonica) wood increases salivary dehydroepiandrosterone sulfate levels after monotonous work. Int J Environ Res Public Health. 14:97. https://doi.org/10.3390/ijerph14010097

12. Kawai E, Nakahara H, Ueda SY, Manabe K, Miyamoto T (2017) A novel approach for evaluating the effects of odor stimulation on dynamic cardiorespiratory functions. PLoS ONE 12:e0172841. https://doi.org/10.1371/ journal.pone.0172841

13. Shen J, Niijima A, Tanida M, Horii Y, Maeda K, Nagai K (2005) Olfactory stimulation with scent of grapefruit oil affects autonomic nerves, lipolysis and appetite in rats. Neurosci Lett 380:289-294. https://doi.org/10.1016/j. neulet.2005.01.058

14. Tanida M, Niijima A, Shen J, Nakamura T, Nagai K (2005) Olfactory stimulation with scent of essential oil of grapefruit affects autonomic neurotransmission and blood pressure. Brain Res 1058:44-55. https://doi. org/10.1016/j.brainres.2005.07.048

15. Tanida M, Niijima A, Shen J, Nakamura T, Nagai K (2006) Olfactory stimulation with scent of lavender oil affects autonomic neurotransmission and blood pressure in rats. Neurosci Lett 398:155-160. https://doi. org/10.1016/j.neulet.2005.12.076

16. Tanida M, Shen J, Niijima A, Yamatodani A, Oishi K, Ishida N, Nagai K (2008) Effects of olfactory stimulations with scents of grapefruit and lavender oils on renal sympathetic nerve and blood pressure in clock mutant mice. Auton Neurosci 139:1-8. https://doi.org/10.1016/j.autne u.2007.12.001

17. Shen J, Niijima A, Tanida M, Horii Y, Maeda K, Nagai K (2005) Olfactory stimulation with scent of lavender oil affects autonomic nerves, lipolysis and appetite in rats. Neurosci Lett 383:188-193. https://doi.org/10.1016/j. neulet.2005.04.010

18. Niijima A, Nagai K (2003) Effect of olfactory stimulation with flavor of grapefruit oil and lemon oil on the activity of sympathetic branch in the white adipose tissue of the epididymis. Exp Biol Med 228:1190-1192 
19. Haze S, Sakai K, Gozu Y (2002) Effects of fragrance inhalation on sympathetic activity in normal adults. Jpn J Pharmacol 90:247-253

20. Sundlof G, Wallin BG (1978) Human muscle nerve sympathetic activity at rest. Relationship to blood pressure and age. J Physiol 274:621-637

21. Vallbo AB, Hagbarth KE, Wallin BG (2004) Microneurography: how the technique developed and its role in the investigation of the sympathetic nervous system. J Appl Physiol 96:1262-1269. https://doi.org/10.1152/ japplphysiol.00470.2003

22. Eckberg DL, Wallin BG, Fagius J, Lundberg L, Torebjork HE (1989) Prospective study of symptoms after human microneurography. Acta Physiol Scand 137:567-569. https://doi.org/10.1111/j.1748-1716.1989.tb08804.x

23. Wallin BG, Esler M, Dorward P, Eisenhofer G, Ferrier C, Westerman R, Jennings $G$ (1992) Simultaneous measurements of cardiac noradrenaline spillover and sympathetic outflow to skeletal muscle in humans. J Physiol 453:45-58

24. Charkoudian N, Joyner MJ, Johnson CP, Eisenach JH, Dietz NM, Wallin BG (2005) Balance between cardiac output and sympathetic nerve activity in resting humans: role in arterial pressure regulation. J Physiol 568:315-321. https://doi.org/10.1113/jphysiol.2005.090076

25. Nagai K, Niijima A, Horii Y, Shen J, Tanida M (2014) Olfactory stimulatory with grapefruit and lavender oils change autonomic nerve activity and physiological function. Auton Neurosci 185:29-35. https://doi. org/10.1016/j.autneu.2014.06.005

26. Vallbo AB, Hagbarth KE, Torebjork HE, Wallin BG (1979) Somatosensory, proprioceptive, and sympathetic activity in human peripheral nerves. Physiol Rev 59:919-957. https://doi.org/10.1152/physrev.1979.59.4.919

27. Sundlof G, Wallin BG (1977) The variability of muscle nerve sympathetic activity in resting recumbent man. J Physiol 272:383-397. https://doi. org/10.1113/jphysiol.1977.sp012050

28. Vieillard S, Peretz I, Gosselin N, Khalfa S, Gagnon L, Bouchard B (2008) Happy, sad, scary and peaceful musical excerpts for research on emotions. Cogn Emot 22:720-752. https://doi.org/10.1080/026999307015035 67

29. Okazaki K, Iwasaki K, Prasad A, Palmer MD, Martini ER, Fu Q, Arbab-Zadeh A, Zhang R, Levine BD (2005) Dose-response relationship of endurance training for autonomic circulatory control in healthy seniors. J Appl Physiol 99:1041-1049. https://doi.org/10.1152/japplphysiol.00085.2005

30. Okada Y, Kamijo Y, Okazaki K, Masuki S, Goto M, Nose H (2009) Pressor responses to isometric biting are evoked by somatosensory receptors in periodontal tissue in humans. J Appl Physiol 107:531-539. https://doi org/10.1152/japplphysiol.91199.2008

31. Kienbaum P, Karlssonn T, Sverrisdottir YB, Elam M, Wallin BG (2001) Two sites for modulation of human sympathetic activity by arterial baroreceptors? J Physiol 531:861-869. https://doi.org/10.1111/j.14697793.2001.0861h.x

32. Munakata M, Aihara A, Imai Y, Abe K, Yoshinaga K (1998) Decreased blood pressure variability at rest in patients with primary aldosteronism. Am J Hypertens 11:828-838. https://doi.org/10.1016/s0895-7061(98)00058-2

33. Rivera AL, Estanol B, Senties-Madrid H, Fossion R, Toledo-Roy JC, Mendoza-Temis J, Morales IO, Landa E, Robles-Cabrera A, Moreno R, Frank A (2016) Heart rate and systolic blood pressure variability in the time domain in patients with recent and long-standing diabetes mellitus. PLoS ONE 11:e0148378. https://doi.org/10.1371/journal.pone.0148378

34. Tanida M, Yamatodani A, Niijima A, Shen J, Todo T, Nagai K (2007) Autonomic and cardiovascular responses to scent stimulation are altered in cry KO mice. Neurosci Lett 413:177-182. https://doi.org/10.1016/j.neule t.2006.11.050

35. Shen J, Niijima A, Tanida M, Horii Y, Nakamura T, Nagai K (2007) Mechanism of changes induced in plasma glycerol by scent stimulation with grapefruit and lavender essential oils. Neurosci Lett 416:241-246. https:// doi.org/10.1016/j.neulet.2006.12.063

36. Satou T, Hayakawa M, Kasuya H, Masuo Y, Koike K (2017) Mouse brain concentrations of -pinene, limonene, linalool, and 1,8-cineole following inhalation. Flavour Fragr J 32:36-39. https://doi.org/10.1002/ff.3342
37. Lochhead JJ, Thorne RG (2012) Intranasal delivery of biologics to the central nervous system. Adv Drug Deliv Rev 64:614-628. https://doi. org/10.1016/j.addr.2011.11.002

38. Chapman CD, Frey WH 2nd, Craft S, Danielyan L, Hallschmid M, Schioth HB, Benedict C (2013) Intranasal treatment of central nervous system dysfunction in humans. Pharm Res 30:2475-2484. https://doi.org/10.1007/ s11095-012-0915-1

39. Pinto JM (2011) Olfaction. Proc Am Thorac Soc 8:46-52. https://doi. org/10.1513/pats.201005-035RN

40. Kettenmann B, Hummel C, Stefan H, Kobal G (1997) Multiple olfactory activity in the human neocortex identified by magnetic source imaging. Chem Senses 22:493-502. https://doi.org/10.1093/chemse/22.5.493

41. Yoder WM, LaRue AK, Rosen JM, Aggarwal S, Shukla RM, Monir J, Smith DW (2014) Evidence of rapid recovery from perceptual odor adaptation using a new stimulus paradigm. Atten Percept Psychophys 76:1093-1105. https://doi.org/10.3758/s13414-013-0620-0

42. Dampney RAL (2017) Resetting of the baroreflex control of sympathetic vasomotor activity during natural behaviors: description and conceptual model of central mechanisms. Front Neurosci 11:461. https://doi. org/10.3389/fnins.2017.00461

43. Hart EC, Charkoudian N, Wallin BG, Curry TB, Eisenach JH, Joyner MJ (2009) Sex differences in sympathetic neural-hemodynamic balance: implications for human blood pressure regulation. Hypertension 53:571-576. https://doi.org/10.1161/hypertensionaha.108.126391

44. Kneale BJ, Chowienczyk PJ, Brett SE, Coltart DJ, Ritter JM (2000) Gender differences in sensitivity to adrenergic agonists of forearm resistance vasculature. J Am Coll Cardiol 36:1233-1238

45. Pluznick JL, Protzko RJ, Gevorgyan H, Peterlin Z, Sipos A, Han J, Brunet I, Wan LX, Rey F, Wang T, Firestein SJ, Yanagisawa M, Gordon Jl, Eichmann A, Peti-Peterdi J, Caplan MJ (2013) Olfactory receptor responding to gut microbiota-derived signals plays a role in renin secretion and blood pressure regulation. Proc Natl Acad Sci USA 110:4410-4415. https://doi. org/10.1073/pnas.1215927110

46. de Siqueira RJ, Rodrigues KM, da Silva MT, Correia Junior CA, Duarte GP, Magalhaes PJ, dos Santos AA, Maia JG, da Cunha PJ, Lahlou S (2014) Linalool-rich rosewood oil induces vago-vagal bradycardic and depressor reflex in rats. Phytother Res 28:42-48. https://doi.org/10.1002/ptr.4953

47. Chrousos GP (2009) Stress and disorders of the stress system. Nat Rev Endocrinol 5:374-381. https://doi.org/10.1038/nrendo.2009.106

48. Takagi C, Nakagawa S, Hirata N, Ohta S, Shimoeda S (2019) Evaluating the effect of aromatherapy on a stress marker in healthy subjects. J Pharm Health Care Sci 5:18. https://doi.org/10.1186/s40780-019-0148-0

49. Fukada M, Kano E, Miyoshi M, Komaki R, Watanabe T (2012) Effect of "rose essential oil" inhalation on stress-induced skin-barrier disruption in rats and humans. Chem Senses 37:347-356. https://doi.org/10.1093/chemse/ bjr108

50. Nakashima T, Akamatsu M, Hatanaka A, Kiyohara T (2004) Attenuation of stress-induced elevations in plasma ACTH level and body temperature in rats by green odor. Physiol Behav 80:481-488. https://doi.org/10.1016/j. physbeh.2003.10.008

51. Tanida M, Katsuyama M, Sakatani K (2008) Effects of fragrance administration on stress-induced prefrontal cortex activity and sebum secretion in the facial skin. Neurosci Lett 432:157-161. https://doi.org/10.1016/j.neule t.2007.12.014

52. Yang S, Zhang L (2004) Glucocorticoids and vascular reactivity. Curr Vasc Pharmacol 2:1-12. https://doi.org/10.2174/1570161043476483

\section{Publisher's Note}

Springer Nature remains neutral with regard to jurisdictional claims in published maps and institutional affiliations. 